The cognitive foundations of early arithmetic skills: it is counting and number judgement, but not finger gnosis, that count

\author{
Imogen Long \\ University College London
}

Stephanie A. Malone

Anne Tolan

Kelly Burgoyne

Michelle Heron-Delaney

Kate Witteveen

Australian Catholic University

Charles Hulme

University College London

Author Note:

This research was in part supported by a grant from the Australian Catholic University.

Correspondence concerning this manuscript should be addressed to Imogen Long, Division of Psychology and Language Sciences, UCL, Chandler House, 2 Wakefield Street, London, WC1N 1PF.

Email: imogen.long.13@ucl.ac.uk

Word count: 3,954 (whole doc including references) 


\begin{abstract}
Following on from ideas developed by Gerstmann, a body of work has suggested that impairments in finger gnosis may be causally related to children's difficulties in learning arithmetic. We report a study with a large sample of typically developing children $(\mathrm{N}=197)$ in which we assess finger gnosis and arithmetic, alongside a range of other relevant cognitive predictors of arithmetic skill (vocabulary, counting, symbolic and nonsymbolic magnitude judgements). Contrary to some earlier claims, we find no meaningful association between finger gnosis and arithmetic skill. Counting and symbolic magnitude comparison are however powerful predictors of arithmetic skill, replicating a number of earlier findings. Our findings seriously question theories that posit either a simple association or a causal connection between finger gnosis and the development of arithmetic skills. (125 words)
\end{abstract}

Key words: arithmetic development, numerical cognition, finger gnosis, symbolic and nonsymbolic magnitude judgement, counting, children 


\section{The cognitive foundations of early arithmetic skills: it is counting and number judgement, but not finger gnosis, that count}

This study investigates the cognitive processes underlying children's arithmetic development. Variations in arithmetic skills appear to be correlated with verbal and counting skills and with tasks deemed to tap an approximate number sense (numerosity judgement tasks) (Bartelet, Vaessen, Blomert, \& Ansari, 2014; Göbel, Watson, Lervåg, \& Hulme, 2014; Mazzocco, Feigenson, \& Halberda, 2011; Moll, Snowling, Göbel, \& Hulme, 2015). Recently, however, claims have been made that sensori-motor abilities, particularly awareness of the fingers (finger gnosis), are related to arithmetic development. Here we assess the claim that finger gnosis is related to the development of arithmetic skills.

Interest in the role of finger gnosis in arithmetic development derives from the work of Gerstmann in the early 1900s who described a syndrome in patients with acquired brain damage involving the co-occurrence of finger agnosia, acalculia, left-right disorientation and handwriting difficulties. He initially argued that a deficit in finger gnosis (a difficulty in distinguishing, naming or recognizing the fingers) may lead to difficulties in arithmetic development due to the close proximity of brain regions involved (a localisation model; Dehaene, Piazza, Pinel, \& Cohen, 2003). He later extended his theory to postulate a functional role of the fingers in learning counting and arithmetic (see, Lebrun, 2005). Although there has been some support for Gerstmann's syndrome in both adults and children, particularly from imaging data (e.g. Mayer et al., 1999; Mazzoni, Pardossi, Cantini, Giorgetti, \& Arena, 1990; Benson \& Gerschwin, 1970) the nature and interpretation of this syndrome remains controversial (e.g. Benton, 1961, 1977; Miller \& Hynd, 2004; Poeck \& Orgass, 1966).

The functional account suggests that the fingers are critical for the development of counting (Butterworth, 1999) and in turn basic calculation skills. This idea has prompted a 
number of studies with children examining the possible role of finger gnosis in the development of counting and arithmetic skills. Noël (2005) used a finger gnosis task in which children were required to identify, without visual assistance, which of their finger(s) were pressed by an examiner, by pointing to the finger(s) that had been touched. The study reported a correlation of $r=.48$ between finger gnosis in Grade 1 and a factor score derived from a set of 6 diverse measures of numerical skills (collection comparison, Arabic digit comparison, subitizing, number writing, addition, and finger counting) obtained 15 months later. The sample size here was small $(\mathrm{N}=41)$ and one clear problem is that the correlation reported does not control for possible effects of age (the children ranged in age from 5 to 7 years of age). Furthermore, the "numerical skills" factor score does not represent a typical or conventional measure of children's arithmetic ability. Penner-Wilger and colleagues (2007) used the same task to assess finger gnosis and found only a weak correlation $(r=.16 ; \mathrm{N}=146)$ with the Woodcock-Johnson calculation subtest (Woodcock, Johnson, \& Mather, 1990).

A study by Wasner, Nuerk, Martignon, Roesch, and Moeller (2016) examined the relationship between finger gnosis and arithmetic in 321 German children in the first year of school (mean age $=6.5$ years). Finger gnosis correlated moderately with both addition and subtraction $(r=.23$ and $r=.24$, respectively). However, in a regression analysis, once other predictors of arithmetic such as age, numerical abilities (magnitude comparison tasks and ordinality judgement) and general cognitive ability (measured via memory testing) had been controlled, finger gnosis predicted only 1-2\% unique variance in arithmetic. This study suggests that any unique relationship between finger gnosis and arithmetic is weak and arguably not of clinical significance.

These recent concurrent correlational studies suggest that finger gnosis is a weaker predictor of arithmetic than previously thought. Longitudinal studies also fail to show any 
convincing evidence of a link between finger gnosis and arithmetic. Fayol, Barrouillet, and Marinthe (1998) found a significant longitudinal relationship between sensori-motor skills (including finger gnosis) at Time 1 and arithmetic 8 months later (partial $r=.36, \mathrm{~N}=189$ ). However, the correlation between finger gnosis and later arithmetic is not reported. Another longitudinal study also failed to find a significant relationship between finger gnosis and calculation skills measured one year later (Penner-Wilger et al., 2009).

To assess the possible causal link between finger gnosis and arithmetic, GraciaBafalluy and Noël (2008) trained 16 5- to 6-year-old children with poor finger gnosis on a number of finger awareness tasks over eight weeks and compared performance in numerical skills to children in a story comprehension control group $(\mathrm{N}=33)$. The study has a number of critical limitations, such as the small sample size and a failure to account for baseline differences between the groups (see also Fischer, 2010). Nevertheless, there appear to be no differences between the finger training and story comprehension groups in counting or addition skills after training. This study has low power but provides no convincing evidence for a causal relationship between finger gnosis and arithmetic.

The relationship between finger gnosis and arithmetic development remains controversial. Many early studies suffer from small sample sizes and a failure to control for known predictors of arithmetic. The present study therefore uses a large sample to examine the relationship between finger gnosis and arithmetic in young typically developing children. We expect that finger gnosis will be a weak concurrent predictor of arithmetic after the effects of age are controlled (Wasner et al., 2016). It should be noted that the finger gnosis test used by Wasner et al. (2016) had low reliability (Cronbach's alpha $=.55)$ which will limit its correlation with other measures. Here we developed a measure of finger gnosis with more trials to ensure adequate reliability. A second aim of this study is to assess the contributions of magnitude 
judgement and counting abilities as predictors of arithmetic. Previous evidence indicates that counting and magnitude judgement tasks are strong predictors of arithmetic (e.g. Göbel et al., 2014; Lyons et al., 2014; Moll, Göbel, \& Snowling, 2015) and we seek to replicate and extend those findings here.

\section{Method}

\section{Participants}

A total of 204 children unselected for ability participated in the study: 130 children in Year $1(\mathrm{n}=77)$ and Year $2(\mathrm{n}=53)$ from two schools in London, England and 74 children in Year 2 from one school in Brisbane, Australia. Mean age was 7 years 1 month (range 5;6-8;8 and $\mathrm{SD}=9.42$ ); children of this age were selected for consistency with previous studies of this issue.

\section{Tests and procedures}

Participants were administered tests of finger gnosis, arithmetic (addition and subtraction), numerical processing (dot counting, symbolic and non-symbolic magnitude comparison) and receptive vocabulary. Children in the UK were also assessed on some other sensori-motor tasks not reported here.

\section{Finger gnosis}

The finger gnosis test assessed a child's ability to identify the finger(s) pressed as in previous studies (e.g. Noël, 2005; Wasner et al., 2016) but included a larger number of trials to increase reliability. Children placed their hands palm down into a box that covered their hands but allowed the experimenter to see them. The experimenter applied light pressure to the child's finger between the nail and finger joint using a stylus. The child pointed to the finger(s) pressed 
using the index finger of the other hand whilst their hands remained out of their sight. There were 25 trials for each hand. The first five involved individual finger presses, the second 10 trials involved two different fingers being pressed in succession and the final 10 trials involved two different fingers being pressed simultaneously. Children were awarded one point for each correct trial (identifying the finger(s) pressed in the correct order). The maximum score on this task was 50.

\section{Arithmetic and number processing tasks}

Children's arithmetic skills were assessed using the Test of Basic Arithmetic and Number Skills (Brigstock, Moll \& Hulme, 2016). Children completed these pencil-and-paper tests in a group setting. For each subtest the children completed three practice tests and were given feedback by the experimenter. They were told to answer as many questions as possible, in the order presented in the booklet and to stop writing when told to do so.

The calculation score was based on the total number of problems (e.g. $3+5$ and $7-3$ ) answered correctly within one minute for each subtest (addition and subtraction). Counting was assessed by a dot counting task in which children had 30 seconds to count the number of dots (2 - 20) in a series of displays and write the associated Arabic numeral. Magnitude judgement tasks involved identifying the larger Arabic digit (symbolic magnitude comparison; digits 1 to 9) or more numerous group of dots (nonsymbolic magnitude comparison; 5 to 57 dots) from a series of stimulus pairs. Children were given 30 seconds for each of these subsets.

\section{Vocabulary}

Receptive vocabulary was assessed by a group-administered test adapted from the British Picture Vocabulary Scale (Dunn \& Dunn, 2009). Four pictures were projected onto the classroom whiteboard and children were asked to identify the picture that matched a target 
word spoken by the experimenter. The items were graded in difficulty and children responded by marking the correct picture (out of four) in a response booklet. There were two practice items (with feedback) followed by 33 test items.

\section{Results}

\section{Descriptive statistics}

Descriptive statistics and reliabilities for all tasks are shown in

Table 1. Calculation was the combined raw score from the addition and subtraction tasks. The finger gnosis test showed good reliability (Cronbach's alpha $=.74$ ) and a normal distribution of scores. All other measures showed a good range of scores without floor or ceiling effects, and have adequate to excellent reliabilities. Preliminary analyses of the data showed no differences between the scores of children in Australia and the UK.

Note that a small minority $(\mathrm{N}=8)$ of children scored 0 on one or more of the following tests: dot counting ( 1 child), magnitude comparison ( 4 children) digit comparison ( 1 child) and vocabulary ( 2 children). It is likely that these children had difficulty following the directions for these group administered tasks. All children were retained in our analyses, but dropping these 8 cases made no difference to the pattern of effects reported. 


\section{Correlations}

The Pearson correlations (and partial correlations controlling for age) between variables are shown in

Table 2. Age was strongly correlated with all the cognitive measures including finger gnosis and calculation ability and for this reason it is critical to control for this in the analyses. Importantly, although finger gnosis correlated with arithmetic ability $(r=.43)$ this is greatly reduced once age was controlled $(r=.12)$. Calculation ability however showed moderate to strong correlations with non-symbolic magnitude comparison $(r=.34)$ symbolic magnitude comparison $(r=.49)$ and dot counting $(r=.58)$ after controlling for age. Unexpectedly, finger gnosis showed a moderate correlation $(r=.38)$ with nonsymbolic magnitude comparison after the effects of age were controlled. 
Table 1

Descriptive Statistics (Means, Standard Deviations, and Range) for all Measures

\begin{tabular}{|c|c|c|c|c|c|}
\hline Variables & $\mathrm{N}$ & Mean (SD) & Reliability & Range & $95 \% \mathrm{CI}$ \\
\hline Finger gnosis $(/ 50)$ & 188 & $31.49(7.95)$ & $.74^{1}$ & $14-50$ & {$[30.35-32.63]$} \\
\hline Single $(/ 10)$ & & $9.41(.97)$ & & $5-10$ & {$[9.27-9.55]$} \\
\hline Successive (/20) & & $10.55(4.07)$ & & $2-20$ & [9.96-11.13] \\
\hline Simultaneous (/20) & & $11.53(4.15)$ & & $2-20$ & [10.94-12.13] \\
\hline Calculation score & 197 & $18.99(11.17)$ & $.89^{2}$ & $0-55$ & [17.43 - 20.56] \\
\hline Addition & & $11.28(7.15)$ & & $0-34$ & [10.27-12.84] \\
\hline Subtraction & & $7.72(4.70)$ & & $0-24$ & [7.06-8.38] \\
\hline
\end{tabular}




\begin{tabular}{lccccc}
\hline Nonsymbolic comparison & 197 & $11.50(7.51)$ & $.72^{2}$ & $0-32$ & {$[10.44-12.55]$} \\
Symbolic comparison & 197 & $19.63(6.78)$ & $.80^{2}$ & $0-38$ & {$[18.68-20.59]$} \\
Dot counting & 197 & $9.65(3.53)$ & $.79^{2}$ & $0-19$ & {$[9.15-10.15]$} \\
Vocabulary (/33) & 197 & $21.04(5.78)$ & $.75^{2}$ & $0-31$ & {$[20.22-21.85]$} \\
& & & & & \\
\hline
\end{tabular}

Notes. ${ }^{1}$ Cronbach's Alpha. ${ }^{2}$ Test-retest reliability. $\mathrm{CI}=$ confidence interval.

Table 2

Correlations among Measures

\begin{tabular}{lcccccc}
\hline \multicolumn{1}{c}{ Measure } & 1 & 2 & 3 & 4 & 5 & 6 \\
\hline 1. Finger gnosis & - & .12 & $.38^{* *}$ & .06 & .10 & .05 \\
2.Calculation score & $.43^{* *}$ & - & $.34^{* *}$ & $.49 * *$ & $.58^{* *}$ & $.24^{*}$ \\
3. Non-symbolic comparison & $.61^{* *}$ & $.61^{* *}$ & - & $.50^{* *}$ & $.36^{* *}$ & $.26^{*}$ \\
4. Symbolic comparison & $.33^{* *}$ & $.62^{* *}$ & $.64^{* *}$ & - & $.46^{* *}$ & $.28^{*}$ \\
5. Dot counting & $.42^{* *}$ & $.73^{* *}$ & $.63^{* *}$ & $.61^{* *}$ & - & $.24^{*}$ \\
6. Vocabulary & $.43^{* *}$ & $.55^{* *}$ & $.63^{* *}$ & $.50^{* *}$ & $.55^{* *}$ & - \\
7. Age & $.58^{* *}$ & $.59^{* *}$ & $.71^{* *}$ & $.47 * *$ & $.63^{* *}$ & $.69^{* *}$
\end{tabular}

Notes. Partial correlations controlling for age are above the diagonal. Simple correlations below. $* p<.05, * * p<.001$. 
Finger gnosis and calculation

The partial correlation between finger gnosis and calculation (controlling for age) was $r=.12,95 \%$ CI $[-0.03,0.26]$ which is a very weak effect that is far from significant even in this large sample (see Figure 1). We also assessed whether finger gnosis might be more closely related to calculation skills in younger than older children. However, a median split of the sample indicated that the partial correlation between finger gnosis and calculation controlling for age was similar in the younger $(r=.13,95 \%$ CI $[-0.09,0.33]$; mean age 77 months, range 66 to 85 months) and older halves of the sample ( $r=.15,95 \%$ CI [-0.05, 0.34]; mean age 93 months, range 86 to 104 months).

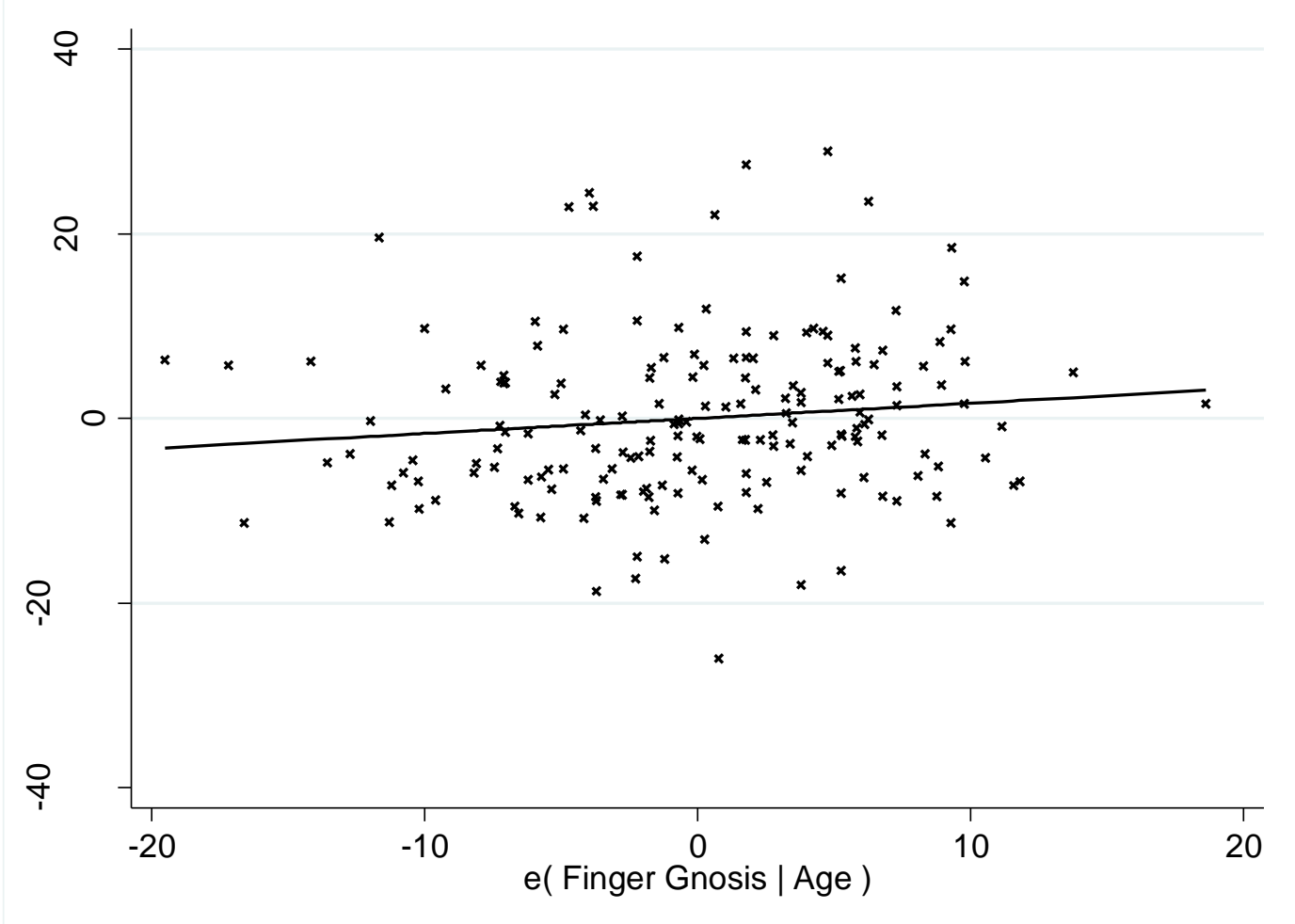

Figure 1. Partial regression leverage plot showing the relationship between arithmetic scores and finger gnosis (controlling for age). 
It is worth noting that the putative role of finger gnosis in the development of arithmetic has often been related to its possible role in the development of counting skills (Butterworth, 1999). However, the partial correlation between finger gnosis and counting controlling for age was also negligible $(r=.10,95 \% \mathrm{CI}[-0.05 ; 0.24])$.

\section{Predictors of calculation ability}

We used multiple regression to model the relationships between calculation ability and the remaining cognitive measures that were significant correlates of calculation after age had been controlled (nonsymbolic magnitude comparison, symbolic magnitude comparison, dot counting and vocabulary). Once age had been controlled $\left(R^{2}=.35 ; p<0.001\right)$ only symbolic magnitude comparison and dot counting were significant unique predictors of calculation (dot counting, unique $R^{2}=.11 ; p<0.001$; symbolic comparison, unique $R^{2}=.04 ; p<0.001$ ); together these two predictors accounted for $26 \%$ of the variance in calculation scores $(p<.001)$. Adding vocabulary and nonsymbolic comparison to the model accounted for no additional variance $\left(R^{2}\right.$ change $\left.=.002 ; p=.35\right)$.

In summary, symbolic magnitude comparison and dot counting were both unique predictors of arithmetic ability (but nonsymbolic magnitude comparison and vocabulary were not). Although finger gnosis was a significant correlate of children's calculation scores this effect is largely attributable to the substantial shared variance between both these measures and age; once the effects of age were controlled the relationship between finger gnosis and calculation ability was negligible, accounting for just $1.4 \%$ of the variance, in line with Wasner et al. (2016).

\section{Discussion}


In this large-scale study we assessed a range of cognitive predictors of variations in arithmetic skills in children. Our particular focus was on the functional theory that posits that there is a possible causal connection between variations in children's finger gnosis and the development of arithmetic skills (e.g. Fayol et al., 1998; Gracia-Bafalluy \& Noël, 2008; Noël, 2005). According to the functional theory this relationship reflects the fact that finger gnosis is critical for the development of counting skills, which are critical for the development of arithmetic skills (e.g. Butterworth, 1999; Rusconi, Walsh, \& Butterworth, 2005). Our results contradict such a theory: we found no evidence for a relationship between finger gnosis and either counting or arithmetic skills after the effects of age were controlled. It is worth emphasising that our measures of finger gnosis, arithmetic and dot counting showed good reliability and a wide range of scores in the age range studied here. Thus, the absence of a relationship between finger gnosis and arithmetic and counting cannot be attributed to a restriction in the range of scores, or to any limitation in the reliability of the measures. In short, this study is well placed to detect possible relationships between finger gnosis and both arithmetic and counting, but none were found. Our results are in line with those from Wasner et al. (2016) who showed that finger gnosis only explained 1-2\% of the variation in arithmetic. The positive effect found in one previous study (Noël, 2005) with a smaller sample likely reflects a failure to take account of age effects. Another study that has claimed to provide support for this theory (Fayol et al., 1998) unfortunately did not separate the effects of finger gnosis from other sensori-motor measures included in the study. A third training study (GraciaBafalluy \& Noël, 2008) had a small sample size, did not control for baseline differences between groups and failed to show any meaningful differences between children in the finger awareness training and control groups in calculation abilities. Taken together, these findings cast doubt on the possible causal relationship between finger gnosis and arithmetic. 
In contrast to these negative findings with respect to finger gnosis, we found that measures of counting and symbolic number judgement (the speed and accuracy of judging which of two Arabic digits represents the larger magnitude) were both independent predictors of variations in children's arithmetic skills. Difficulties in learning to count are a prominent deficit in children with Mathematics Disorder (Geary, Bow-Thomas, \& Yao, 1992; Gersten, Jordan, \& Flojo, 2005) and there is good reason to suppose that the development of counting skills is one crucial foundation for the development of arithmetic in children (Bartelet et al., 2014; Moll et al., 2015b). Our study provides further support for a close link between variations in children's counting skills and the development of arithmetic skills (assessed here by the efficiency of basic addition and subtraction skills).

Our study also found a link between variations in children's symbolic magnitude judgements and arithmetic development. The symbolic magnitude judgement task requires knowledge of Arabic digits and the magnitudes they represent. Knowledge of Arabic digits is clearly a critical prerequisite for learning to perform written arithmetic (arguably analogous to the role of letter-sound knowledge in learning to read). Mastery of reading and arithmetic both depend upon learning the symbol set used (letters and digits). Importantly, nonsymbolic magnitude judgement here was a weaker correlate of arithmetic skill than symbolic magnitude judgement, in line with recent meta-analyses (De Smedt, Noël, Gilmore, \& Ansari, 2013; Schneider et al., 2016). This relates to the theoretical issue of the possible role of an approximate number sense in arithmetic development (see Dehaene, 2011). The current data suggest that variations in verbal processes (including counting and knowledge of Arabic digits) may be more closely related to the development of arithmetic than variations in nonsymbolic (nonverbal) magnitude processing (see also Göbel et al., 2014; Lyons et al., 2014). 
One interesting and unexpected finding from the present study was that finger gnosis correlated moderately to strongly with nonsymbolic magnitude judgement skills ( $r=.61$; after age is controlled $r=.38$ ). This is a finding that deserves replication. It is hard to think of a functional relationship between finger gnosis and magnitude judgement performance, and we would speculate that this effect may reflect the anatomical overlap between the brain systems in the parietal lobe responsible for numerosity encoding and sensori-motor control. Alternatively, a third variable, inhibitory control, may be driving this relationship as it has been shown to correlate with nonsymbolic magnitude comparison (Gilmore, et al., 2013) which, speculatively, may also be involved in finger awareness. Further studies are required to test these ideas.

One limitation of the present study is that it examines children who have generally mastered basic addition and subtraction skills, and therefore may be too old to identify any relationship that may exist in younger children. Children of this age-range were chosen to match previous studies, but further research is needed to examine this relationship in children at the beginning of formal education, who will have weaker counting and numerical abilities.

In summary, our study provides no support for that the idea that finger gnosis is related in any important way to arithmetic development in children. There is, however, clear evidence to support a close relationship between both counting and symbolic magnitude judgement and variations in arithmetic abilities. Such effects provide further evidence that variations in verbal processes (including counting and knowledge of Arabic digits) are closely related to the development of arithmetic skills (see also Göbel et al., 2014; Moll et al., 2015b) 


\section{Acknowledgements}

Part of this study was supported by a grant from the Australian Catholic University. We would like to thank the schools and children who participated in the study. The authors have no conflicts of interests arising from the publication of this study.

\section{References}

Bartelet, D., Vaessen, A., Blomert, L., \& Ansari, D. (2014). What basic number processing measures in kindergarten explain unique variability in first-grade arithmetic proficiency? Journal of Experimental Child Psychology, 117, 12-28. doi:10.1016/j.jecp.2013.08.010

Benson, D. F., \& Geshwind, N. (1970). Developmental Gerstmann's syndrome. Neurology, 20(3), 293-298

Benton, A. L. (1961). The fiction of the "Gerstmann Syndrome". Journal of Neurology, Neurosurgery, and Psychiatry, 24(2), 176-181. doi:10.1016/0093-934X(77)90005-0

Benton, A. L. (1977). Reflections on the Gerstmann syndrome. Brain and Language, 4(1), $45-62$. 
Brigstock, S., Moll, K., \& Hulme, C. (2016). TOBANS: Test of basic arithmetic and numeracy skills. Retrieved from: https://global.oup.com/education/content/primary/series/tobans/?region=uk

Butterworth, B. (2000). The mathematical brain. London: Macmillan

De Smedt, B., Noël, M. P., Gilmore, C., \& Ansari, D. (2013). How do symbolic and nonsymbolic numerical magnitude processing skills relate to individual differences in children's mathematical skills? A review of evidence from brain and behavior. Trends in Neuroscience and Education, 2(2), 48-55. doi:10.1016/j.tine.2013.06.001

Dehaene, S. (2011). The number sense: How the mind creates mathematics. Revised and updated edition. USA: Oxford University Press

Dunn, L. M., \& Dunn, D. M. (2009). The British picture vocabulary scale. UK:GL Assessment

Fayol, M., Barrouillet, P., \& Marinthe, C. (1998). Predicting arithmetical achievement from neuro-psychological performance: A longitudinal study. Cognition, 68(2). doi:10.1016/S0010-0277(98)00046-8

Fischer, J. P. (2010). Numerical performance increased by finger training: A fallacy due to regression toward the mean? Cortex, 46(2), 272-273. doi:10.1016/j.cortex.2008.06.010

Geary, D. C., Bow-Thomas, C. C., \& Yao, Y. (1992). Counting knowledge and skill in cognitive addition: A comparison of normal and mathematically disabled children. Journal of Experimental Child Psychology, 54(3), 372-391. doi:10.1016/0022-0965(92)90026-3

Gersten, R., Jordan, N. C., \& Flojo, J. R. (2005). Early Identification and Interventions for Students With Mathematics Difficulties. Journal of Learning Disabilities, 38(4), 293304. doi: $10.1177 / 00222194050380040301$ 
Gilmore, C. Attridge, N., Clayton, S., Cragg, L., Johnson, S., Marlow, N., Simms, V., \& Inglis, M. (2013). Individual differences in inhibitory control, not non-verbal number acuity, correlate with mathematics achievement. PloS One, 8(6). doi:10.1371/journal.pone.0067374

Göbel, S. M., Watson, S. E., Lervåg, A., \& Hulme, C. (2014). Children’s arithmetic development: It is number knowledge, not the approximate number sense, that counts. Psychological Science. doi:10.1177/0956797613516471

Gracia-Bafalluy, M., \& Noël, M. P. (2008). Does finger training increase young children's numerical performance? Cortex, 44(4), 368-375. doi:10.1016/j.cortex.2007.08.020

Lebrun, Y. (2005). Gerstmann's Syndrome. Journal of Neurolinguistics, 18(4), 317-326. doi:10.1016/j.jneuroling.2004.11.010

Lyons, I. M., Price, G. R., Vaessen, A., Blomert, L., \& Ansari, D. (2014). Numerical predictors of arithmetic success in grades 1-6. Developmental Science, 17(5), 714726. doi:10.1111/desc. 12152

Mayer, E., Martory, M. D., Pegna, A. J., Landis, T., Delavelle, J., \& Annoni, J. M. (1999). A pure case of Gerstmann syndrome with a subangular lesion. Brain, 122(6), 11071120. doi:10.1093/brain/122.6.1107

Mazzocco, M. M., Feigenson, L., \& Halberda, J. (2011). Preschoolers’ precision of the approximate number system predicts later school mathematics performance. PLoS One, 6(9). doi:10.1371/journal.pone.0023749

Mazzoni, M., Pardossi, L., Cantini, R., Giorgetti, V., \& Arena, R. (1990). Gerstmann Syndrome: A Case Report. Cortex, 26(3), 459-467. doi:10.1016/S0010-9452(13)80096-2 
Miller, C. J., \& Hynd, G. W. (2004). What ever happened to developmental Gerstmann's syndrome? Links to other paediatric, genetic, and neurodevelopmental syndromes. Journal of Child Neurology, 19(4), 282-289. doi:10.1177/088307380401900408

Moll, K., Göbel, S. M., \& Snowling, M. J. (2015a). Basic number processing in children with specific learning disorders: Comorbidity of reading and mathematics disorders. Child Neuropsychology, 21(3), 399-417. doi:10.1080/09297049.2014.899570

Moll, K., Snowling, M. J., Göbel, S. M., \& Hulme, C. (2015b). Early language and executive skills predict variations in number and arithmetic skills in children at family-risk of dyslexia and typically developing controls. Learning and Instruction, 38, 53-62. doi:10.1016/j.learninstruc.2015.03.004

Noël, M. P. (2005). Finger gnosia: A predictor of numerical abilities in children? Child Neuropsychology, 11(5), 413-430. doi:10.1080/09297040590951550

Penner-Wilger, M., Fast, L., LeFevre, J.-A., Smith-Chant, B. L., Skwarchuk, S., Kamawar, D., \& Bisanz, J. (2007). The foundations of numeracy: Subitizing, finger gnosia, and fine-motor ability. In Proceedings of the 29th annual cognitive science society (pp. 1385-1390). Cognitive Science Society Austin, TX. Retrieved from http://csjarchive.cogsci.rpi.edu/proceedings/2007/docs/p1385.pdf

Penner-Wilger, M., Fast, L., LeFevre, J., Smith-Chant, B. L., Skwarchuk, S., Kamawar, D., \& Bisanz, J. (2009). Subitizing, finger gnosis, and the representation of number. Proceedings of the 31st Annual Cognitive Science Society, 520-525.

Poeck, K., \& Orgass, B. (1966). Gerstmann's Syndrome and Aphasia. Cortex, 2(4), 421-437. doi:10.1016/S0010-9452(66)80018-7

Rusconi, E., Walsh, V., \& Butterworth, B. (2005). Dexterity with numbers: rTMS over left angular gyrus disrupts finger gnosis and number processing. Neuropsychologia, 43(11), 1609-1624. doi:10.1016/j.neuropsychologia.2005.01.009 
Schneider, M., Beers, K., Coban, L., Merz, S., Susan Schmidt, S., Stricker, J., \& De Smedt, B. (2016). Associations of non-symbolic and symbolic numerical magnitude processing with mathematical competence: a meta-analysis. Developmental Science. doi:10.1111/desc. 12372

Wasner, M., Nuerk, H.-C., Martignon, L., Roesch, S., \& Moeller, K. (2016). Finger gnosis predicts a unique but small part of variance in initial arithmetic performance. Journal of Experimental Child Psychology, 146, 1-16. doi:10.1016/j.jecp.2016.01.006

Woodcock, R. W., Johnson, M. B., \& Mather, N. (1990). Woodcock-Johnson psychoeducational battery - Revised. DLM Teaching Resources. 